\title{
Typing of methicillin-resistant Staphylococcus aureus by antibiotic resistance phenotypes
}

\author{
M.T. GILLESPIE*, B. R.LYON† and R.A. SKURRAY \\ Department of Microbiology, Monash University, Clayton, Victoria 3168, Australia
}

\begin{abstract}
Summary. The identification of new epidemic strains of methicillin-resistant Staphylococcus aureus is essential for rapid, effective infection control. We have developed a typing method which uses antibiotic sensitivity patterns to differentiate methicillin-resistant $S$. aureus and which is faster and more cost-effective than biochemical analysis or bacteriophage typing. Characterisation of phenotypes which are chromosomally-encoded, plasmid- or chromosomally-encoded or exclusively plasmid-mediated has enabled us to separate Australian strains of methicillinresistant $S$. aureus into 11 classes, representatives of which were indistinguishable by bacteriophage type, or plasmid profile alone. The value of this procedure is thus clearly shown.
\end{abstract}

\section{Introduction}

With the increased spread of methicillin-resistant Staphylococcus aureus in Australia, Britain and the USA (Lyon and Skurray, 1987), microbiologists have sought typing systems which distinguish new epidemic strains from endemic ones. New $S$. aureus strains are usually detected by their different pattern of antibiotic resistance. The spread of these strains may be monitored by antibiograms, biochemical tests or by bacteriophage typing. Many methicillinresistant $S$. aureus strains are not typable by bacteriophages of the International Typing Set; supplementary bacteriophages have been isolated to distinguish these non-typable strains of $S$. aureus (Vickery et al., 1983; Richardson et al., 1988).

Recently, electrophoretic separation of cellular proteins has been employed to differentiate methicillin-resistant $S$. aureus (Gaston et al., 1988). Coomassie Blue staining of whole-cell extracts has not been reproducible or has failed to distinguish $S$. aureus strains which could be differentiated by their susceptibility to bacteriophages of the International Set. In contrast, other electrophoretic methods such as Western blot analysis of culture supernate and analysis of cellular proteins with ${ }^{35} \mathrm{~S}$ -

Received 30 Jan. 1989; revised version accepted 3 Aug. 1989.

* Present address: St Vincent's Institute of Medical Research, 9 Princes Street, Fitzroy, Victoria 3065, Australia.

† Present address: C.S.I.R.O. Division of Plant Industry, Canberra, A.C.T. 2601, Australia.

‡ Correspondence should be sent to Dr R. A. Skurray, Department of Microbiology, Monash University, Clayton, Victoria 3168, Australia. methionine have distinguished outbreak strains from epidemic strains (Krikler et al., 1986; Stephenson et al., 1986; Gaston et al., 1988; Lee and Burnie, 1988).

Plasmid typing has been used successfully to trace strains prevalent in the USA (Kozarsky et al., 1986; Rhinehart et al., 1987) and in Ireland (Coleman et al., 1985). We have used plasmid profiles to monitor the spread of epidemic strains of methicillin-resistant $S$. aureus throughout Australia (Lyon et al., 1983, 1984a). This procedure, combined with restriction endonuclease analysis, DNA hybridisation and antibiogram results, has identified the locality of encodement (chromosomeor plasmid-mediated) of antimicrobial resistance determinants in more than 2000 strains isolated in hospitals throughout Australia. These results supported a clonal origin for Australian methicillinresistant $S$. aureus since isolates had common chromosomally-encoded resistance phenotypes but differed in their plasmid content. The typing scheme described here was adapted to distinguish strains of methicillin-resistant $S$. aureus on the basis of their plasmid-encoded phenotypes and will differentiate current strains of epidemic methicillinresistant $S$. aureus (EMRSA) from other methicillin-resistant strains detected throughout the late 1960 s.

\section{Materials and methods}

\section{Strains of Staphylococcus aureus}

Bacterial strains were obtained from Australian hos- 
pitals between 1946 and 1982 and many have undergone preliminary analysis (Lyon et al., 1983, 1984a; Gillespie et al., 1985, 1986a); relevant strains and plasmids are listed in tables I and II.

\section{Antibiotic sensitivity testing}

L-broth and L-agar media, and methods for the determination of antimicrobial and heavy metal resistance and penicillinase production by use of multipoint inoculation were described by Lyon et al. $(1983,1984 a)$. An overnight bacterial suspension was diluted so that approximately $10^{4} \mathrm{cfu} /$ inoculum site were delivered on to the surface of an L-agar plate. Plates were incubated at $37^{\circ} \mathrm{C}$ for $24 \mathrm{~h}$. Antimicrobial agents were incorporated into L-broth and L-agar at the following concentrations: acriflavine (Ac; May and Baker, Australia), $100 \mu \mathrm{g} / \mathrm{ml}$ (low-level) and $250 \mu \mathrm{g} / \mathrm{ml}$ (high-level); amikacin (Ak; Bristol Laboratories, USA), $8 \mu \mathrm{g} / \mathrm{ml}$; ampicillin (Ap; CSL, Australia), $10 \mu \mathrm{g} / \mathrm{ml}$; benzalkonium chloride (Bc; Sigma Chemical Co., USA), $2 \mu \mathrm{g} / \mathrm{ml}$; cadmium nitrate (Cd), $5 \times 10^{-5} \mathrm{M}$; cetyltrimethylammonium bromide $(\mathrm{Ct}$; BDH Chemicals, England), $1 \mu \mathrm{g} / \mathrm{ml}$; chloramphenicol ( $\mathrm{Cm}$; Boehringer Mannheim, West Germany), $16 \mu \mathrm{g} / \mathrm{ml}$; clindamycin ( $\mathrm{Cl}$; The Upjohn Co, USA), $10 \mu \mathrm{g} / \mathrm{ml} ; 4^{\prime} 4^{\prime}$ diamidinodiphenylamine dihydrochloride (Dd; May and Baker, Australia), $50 \mu \mathrm{g} / \mathrm{ml}$; erythromycin (Em; Abbott Laboratories, Australia), $5 \mu \mathrm{g} / \mathrm{ml}$; ethidium bromide (Eb; Sigma Chemical Co., USA), $20 \mu \mathrm{g} / \mathrm{ml}$ (low-level) and $100 \mu \mathrm{g} / \mathrm{ml}$ (high-level); fusidic acid (Fa; Leo Pharma- ceuticals, Denmark), $1 \mu \mathrm{g} / \mathrm{ml}$; gentamicin (Gm; Schering Corp., USA), $4 \mu \mathrm{g} / \mathrm{ml}$ (low-level) and $500 \mu \mathrm{g} / \mathrm{ml}$ (highlevel); kanamycin ( $\mathrm{Km}$; Bristol Laboratories, USA), $10 \mu \mathrm{g} / \mathrm{ml}$ (low-level) and $500 \mu \mathrm{g} / \mathrm{ml}$ (high-level); mercuric nitrate $(\mathrm{Hg}), 1 \times 10^{-5} \mathrm{M}$; methicillin (Mc; Beecham Australia), $8 \mu \mathrm{g} / \mathrm{ml}$; neomycin ( $\mathrm{Nm}$; The Upjohn Co., USA), $10 \mu \mathrm{g} / \mathrm{ml}$ (low-level) and $500 \mu \mathrm{g} / \mathrm{ml}$ (high-level); paromomycin (Pm; Warner Lambert Co., USA), $8 \mu \mathrm{g}$ / $\mathrm{ml}$; penicillin G (Pc, Glaxo, Australia) $10 \mu \mathrm{g} / \mathrm{ml}$; propamidine isethionate ( $\mathrm{Pi}$; May and Baker, Australia), $50 \mu \mathrm{g} /$ $\mathrm{ml}$; rifampicin (Rf; Sigma Chemical Co., USA), $1 \mu \mathrm{g} /$ $\mathrm{ml}$; sodium arsenate (Asa), $7 \times 10^{-5} \mathrm{M}$; sodium arsenite (Asi), $4 \times 10^{-4} \mathrm{M}$; spectinomycin (Sp; The Upjohn Co., USA), $50 \mu \mathrm{g} / \mathrm{ml}$; streptomycin (Sm; Glaxo, Australia), $10 \mu \mathrm{g} / \mathrm{ml}$; tetracycline (Tc; Sigma Chemical Co., USA), $5 \mu \mathrm{g} / \mathrm{ml}$; tobramycin (Tm; Eli Lilly \& Co., USA), $4 \mu \mathrm{g} /$ $\mathrm{ml}$ (low-level) and $500 \mu \mathrm{g} / \mathrm{ml}$ (high-level); trimethoprim (Tp; Sigma Chemical Co., USA), $25 \mu \mathrm{g} / \mathrm{ml}$ (low-level) and $500 \mu \mathrm{g} / \mathrm{ml}$ (high-level); vancomycin (Vm; Eli Lilly \& Co., USA), $4 \mu \mathrm{g} / \mathrm{ml}$.

Appropriate care was taken when handling compounds such as cadmium nitrate, mercuric nitrate, sodium arsenate and sodium nitrite as well as the potent mutagen ethidium bromide; protective gloves were worn when handling these agents and they were used only in an exhaust-ventilated safety cabinet. In addition, these agents had to be added to molten agar at a temperature low enough to minimise vaporisation (i.e., $50^{\circ} \mathrm{C}$ ). Procedures for the detoxification of ethidium bromide have been described by Lunn and Sansome (1987).

Table I. Characteristics of $S$. aureus plasmids

\begin{tabular}{|c|c|c|c|c|}
\hline Plasmid & Resistance to* & $\begin{array}{l}\text { Size } \\
(\mathrm{kb})\end{array}$ & $\begin{array}{c}\beta \text {-lactamase } \\
\text { production }\end{array}$ & Reference \\
\hline pSK1 & GmTmKmAcEbQaDdPiTp & $28 \cdot 4$ & - & Gillespie et al., $1987 b$ \\
\hline pSK2 & $\mathrm{Cm}$ & $4 \cdot 5$ & - & Gillespie et al., $1987 b$ \\
\hline pSK3 & $\ldots$ & $1 \cdot 5$ & - & Lyon et al., 1984a \\
\hline pSK4 & GmTmKmAcEbQaDdPiTpPc & $35 \cdot 1$ & + & Gillespie et al., $1987 b$ \\
\hline pSK 7 & AcEbQaDdPiTp & $23 \cdot 7$ & - & Gillespie et al., $1987 b$ \\
\hline pSK8 & GmTmKmAcEbQaDdPiTpPc & $35 \cdot 1$ & + & Gillespie et al., $1987 b$ \\
\hline pSK 9 & GmTmKmAcEbQaDdPi & $25 \cdot 7$ & - & Gillespie et al., $1987 b$ \\
\hline pSK11 & GmTmKmTpPc & $41 \cdot 8$ & + & Gillespie et al., $1987 b$ \\
\hline pSK 14 & GmTmKmAcEbQaDdPi & $24 \cdot 4$ & - & Gillespie et al., $1987 b$ \\
\hline pSK 15 & GmTmKmAcEbQaDdPiPc & $31 \cdot 1$ & + & Gillespie et al., $1987 b$ \\
\hline pSK16 & GmTmKmAcEbQaDdPiTpPc & $35 \cdot 1$ & + & Gillespie et al., $1987 b$ \\
\hline pSK 17 & GmTmKmAcEbQaDdPiTpPc & $36 \cdot 4$ & + & Gillespie et al., $1987 b$ \\
\hline pSK 18 & AcEbQaDdPi & $19 \cdot 7$ & - & Lyon et al., $1984 a$ \\
\hline pSK21 & $\mathrm{AcEbQaCdHg}$ & $35 \cdot 3$ & - & \\
\hline pSK23 & $\mathrm{GmTmKmAcEbQaCdHg}$ & $38 \cdot 0$ & - & Lyon and Skurray, 1987 \\
\hline pSK41 & GmTmKmNmPmAkEbQa & $47 \cdot 8$ & - & Lyon et al., 1987 \\
\hline pSK 51 & PcAsaAsiCdHg & $29 \cdot 1$ & + & Gillespie et al., 1984 \\
\hline pSK52 & Tc & $4 \cdot 4$ & - & Gillespie et al., $1986 b$ \\
\hline pSK57 & AcEbQaDdPiPcCdHg & $28 \cdot 8$ & + & Gillespie et al., 1986a \\
\hline pSK 58 & $\ldots$ & $2 \cdot 4$ & - & Gillespie et al., 1984 \\
\hline pSK 89 & EbQa & $2 \cdot 4$ & - & Lyon and Skurray, 1987 \\
\hline pSK90 & $\mathrm{Cm}$ & $4 \cdot 6$ & - & Gillespie and Skurray, 1988 \\
\hline pUW3626 & GmTmKmNmPmAkEbQaPc & $54 \cdot 4$ & + & Lyon et al., 1987 \\
\hline
\end{tabular}

* See Materials and methods for abbreviations. 
Table II. Characteristics of $S$. aureus strains

\begin{tabular}{|c|c|c|c|}
\hline Strain & Resistant to* & Plasmids carried & Reference \\
\hline SK20 & PcMcSmTcCdHgEmClSp & pSK3 & \\
\hline SK413 & PcMcSmTcCdHgEmCiSpGmTmKmNmPmAcEbQaTp ${ }^{L}$ & pSK21 & \\
\hline SK 430 & PcMcSmTcCdHgEmCiSpAcEbQaDdPiTp ${ }^{\mathrm{L}}$ & pSK 18 & Tennent et al., 1985 \\
\hline SK445 & PcMcSmTcCdHgEmCiSpGmTmKmNmPmAkEbQa & pUW3626 & Lyon et al., 1987 \\
\hline SK449 & $\mathrm{PcMcSmTcCdHgAcEbQaDdPi}$ & pSK52, pSK57 & Gillespie et al., $1986 a$ \\
\hline SK456 & PcMcSmTcCdHgEmClSpGmTmKmAcEbQaDdPiTp ${ }^{H}$ & pSK 4 & Lyon et al., $1984 a$ \\
\hline SK457 & PcMcSmTcCdHgEmCISpGmTmKmTp ${ }^{\mathrm{L}}$ & & Gillespie et al., 1984 \\
\hline SK 460 & PcMcSmTcCdHgEmClSpGmTmKmNmPmAkAcEbQaDdPiTp ${ }^{\mathrm{H}}$ & pSK 3 , pSK 7 & Lyon et al., $1984 a$ \\
\hline SK480 & PcMcSmTcCdHgEmClSpGmTmKmAcEbQaDdPiTp ${ }^{\mathrm{H}} \mathrm{CmFa}$ & pSK2, pSK 3, pSK 8 & Lyon et al., 1983 \\
\hline SK501 & PcMcSmTcCdHgEmClSpGmTmKmAcEbQaDdPiTp ${ }^{\mathrm{H}} \mathrm{Cm}$ & pSK2, pSK 3, pSK 11 & \\
\hline SK529 & PcMcSmTcCdHgEmClSpGmTmKmAcEbQaDdPiTp ${ }^{\mathrm{H}} \mathrm{Cm}$ & $\mathrm{pSK} 1, \mathrm{pSK} 2, \mathrm{pSK} 3$ & Lyon et al., $1984 a$ \\
\hline SK565 & PcMcSmTcCdHgEmClSpGmTmKmAcEbQaDdPiTp ${ }^{\mathbf{L}} \mathrm{Cm}$ & pSK2, pSK3, pSK 15 & Lyon et al., $1984 a$ \\
\hline SK604 & PcMcSmTcCdHgEmClSpGmTmKmAcEbQaDdPiTp $^{\mathbf{L} C m}$ & pSK 2 , pSK 9 & Lyon et al., $1984 a$ \\
\hline SK605 & PcMcSmTcCdHgEmClSpGmTmKmNmPmAkTp ${ }^{\mathrm{L}}$ & & \\
\hline SK612 & PcMcSmTcCdHgEmClSpGmTmKmNmPmAkEbQa & pSK41 & Lyon et al., 1987 \\
\hline SK634 & PcMcSmTcCdHgEmClSpGmTmKmAcEbQaDdPiTp ${ }^{\mathrm{H}}$ & pSK3, pSK 16 & Lyon et al., $1984 a$ \\
\hline SK654 & PcMcSmTcCdHgEmClSpGmTmKmNmPmAcEbQaTp & pSK 23 & \\
\hline SK656 & PcMcSmTcCdHgEmClSpGmTmKmAcEbQaDdPiTp ${ }^{L} \mathrm{Cm}$ & pSK2, pSK 3, pSK14 & Lyon et al., $1984 a$ \\
\hline SK 707 & PcMcSmTcCdHgEmClSpGmTmKmAcEbQaDdPiTp ${ }^{\mathrm{H}}$ & pSK3, pSK 17 & \\
\hline SK 1448 & PcSmTcAsaAsiCdHgEbQa & pSK 89 & \\
\hline SK 1651 & PcMcSmTcCdHgAsaAsiCm & $\begin{array}{l}\text { pSK51, pSK52 } \\
\text { pSK } 58, \text { pSK } 90\end{array}$ & Gillespie et al., 1984 \\
\hline
\end{tabular}

* See Materials and methods for abbreviations.

\section{Results and discussion}

\section{Differentiation of methicillin-resistant S. aureus}

Genetic analysis of $S$. aureus strains from Australian hospitals has shown that the outbreaks of methicillin (Mc)-resistant $S$. aureus seen after 1980 resulted from the spread of a single epidemic strain or closely-related derivatives of that strain (Lyon et al., 1984a; Skurray et al., 1988). All of these isolates were resistant to penicillin $(\mathrm{Pc})$, streptomycin $(\mathrm{Sm})$ and tetracycline (Tc), and $95 \%$ were resistant to erythromycin (Em) and spectinomycin (Sp). They differed, however, in their susceptibility to the aminoglycosides amikacin (Ak), gentamicin (Gm), kanamycin $(\mathrm{Km})$, neomycin $(\mathrm{Nm})$ and tobramycin $(\mathrm{Tm})$. Resistance to $\mathrm{Gm}$, $\mathrm{Km}$ and $\mathrm{Tm}$ was shown to be encoded by a $4 \cdot 7-\mathrm{kb}$ transposon, Tn4001, which could occupy either a plasmid or a chromosomal locus (Lyon et al., 1984b, 1987; Gillespie et al., 1987b). Tn4001 was most frequently ( $77 \%$ of $\mathrm{Gm}^{\mathrm{r}}$ isolates) associated with one of a group of structurally-related plasmids (known as the pSK1 family), $24-42 \mathrm{~kb}$ in size, which may confer resistance to a high level of trimethoprim (Tp), to antiseptic agents such as acriflavine (Ac) and quaternary ammonium compounds $(\mathrm{Qa}$; e.g., benzalkonium chloride, $(\mathrm{Bc})$, cetyltrimethylammonium chloride $(\mathrm{Ct})$ ) and to ethidium bromide (Eb) (Gillespie et al., 1987b; Skurray et al., 1988). The last-named phenotypes are generally plasmid-mediated; strains which harbour such plasmids are easily identified, a fact which forms the basis of the following typing scheme.

Since all the strains of methicillin-resistant $S$. aureus examined encoded resistance to $\mathrm{Pc}, \mathrm{Sm}$ and Tc and the heavy metals cadmium (Cd) and mercury (Hg) (table II), this was considered the reference point for the identification system (figure). $\mathrm{The} \mathrm{Hg}$, $\mathrm{Mc}$ and Tc resistance determinants have been mapped in close association on the chromosomes of a number of isolates of methicillin-resistant $S$. aureus by hybridisation and molecular cloning analyses (Gillespie et al., 1987a; Matthews et al., 1987), thereby strengthening the claim for a clonal origin of methicillin-resistant strains of $S$. aureus. One feature that distinguished methicillin-resistant isolates detected after 1970 from those detected prior to this date was their susceptibility to arsenate (Asa) and arsenite (Asi). Methicillin-resistant $S$. aureus strains isolated in the 1960 s frequently possessed heavy-metal resistance ( $\beta$-lactamase or penicillinase) plasmids which mediated resistance to Pc, Asa, Asi, Cd and $\mathrm{Hg}$, whereas the majority $(>99 \%$ ) of methicillin-resistant isolates after 1970 




Figure. Steps involved in the differentiation of methicillin-resistant $S$. aureus on the basis of antibiotic resistance phenotypes. Representative strains along with their plasmids are indicated. Abbreviations are as in the text.

did not carry such a plasmid (Gillespie et al., 1985); in strains isolated after 1970 that carried a heavymetal resistance plasmid, the plasmid did not confer $\mathrm{Asa}^{\mathrm{T}}$ or $\mathrm{Asi}^{\mathrm{r}}$.

Once the methicillin-resistant isolate has been shown to be of the post-1970s type, the strains can be divided by phenotypes which were found to be plasmid-encoded in isolates from the 1980s (see figure). The first such differentiating phenotype is that of resistance towards $\mathrm{Ac}, \mathrm{Eb}$ and $\mathrm{Qa}$. Resistant strains fell into one of two classes; those with highlevel resistance possessed minimum inhibitory concentrations (MICs) of $340,6,4$ and $180 \mu \mathrm{g} / \mathrm{ml}$ for $\mathrm{Ac}, \mathrm{Bc}, \mathrm{Ct}$ and $\mathrm{Eb}$, whereas those with low-level resistance demonstrated MICs of 6,4 and $30 \mu \mathrm{g} /$ $\mathrm{ml}$, for $\mathrm{Bc}, \mathrm{Ct}$ and $\mathrm{Eb}$, respectively, but were sensitive to Ac. This point effectively differentiated the strains by their plasmid complement into those which carried (i) a pSK1-type or heavy-metal resistance plasmid (high-level), or (ii) a selftransmissible plasmid (low-level), such as pSK 41 or pUW3626 (Cohen et al., 1982; Lyon et al., 1987), or (iii) neither plasmid type (sensitive strain); the only exception to this was a strain carrying pSK 11 , a pSK1-family plasmid, which did not express resistance to $\mathrm{Ac}, \mathrm{Eb}$ or $\mathrm{Qa}$ (see below).

\section{Strains sensitive to acriflavine, ethidium bromide and to quaternary ammonium compounds}

Strains sensitive to AcEbQa may be further differentiated by their ability to encode gentamicin resistance $\left(\mathrm{Gm}^{\mathrm{r}}\right)$ (figure). Strains which are sensitive to $\mathrm{Gm}$, such as SK20 (table II and figure), do not possess a large molecular weight plasmid species and may be devoid of extrachromosomal DNA. Such strains represent the "core" strain of contemporary Australian isolates since all resistance determinants are chromosomally-determined. As with $\mathrm{Ac}^{\mathrm{r}} \mathrm{Eb}^{\mathrm{r}} \mathrm{Qa}^{\mathrm{r}}$ strains, resistance to $\mathrm{Gm}$ can be of two levels-high-level (MIC $>4096 \mu \mathrm{g} / \mathrm{ml}$ ) and low-level (MIC $32 \mu \mathrm{g} / \mathrm{ml}$ ). High-level resistant strains, such as SK605 (table II and figure), were additionally resistant to high levels of $\mathrm{Tm}, \mathrm{Km}$, 
$\mathrm{Nm}$ and Ak (MICs > 4096, 256-4096, 2048 and $512 \mu \mathrm{g} / \mathrm{ml}$, respectively); this high level of resistance is unlikely to be due to a novel aminoglycoside modifying enzyme, but is more probably attributable to a chromosomal mutation leading to altered cell-wall permeability (Gillespie et al., 1984). The low-level $\mathrm{Gm}^{\mathrm{r}}$ phenotype is encoded by $\mathrm{Tn} 4001$ which additionally confers resistance to tobramycin (Tm) and kanamycin (Km) (MICs 64 and $256 \mu \mathrm{g} /$ $\mathrm{ml}$; Lyon et al., 1987). Strains with low-level $\mathrm{Gm}^{\mathrm{r}}$ (MIC $128 \mu \mathrm{g} / \mathrm{ml}$ ) can be further subdivided on the basis of their susceptibility to trimethoprim (Tp). High-level $\mathrm{Tp}^{\mathrm{r}}$ strains (MIC $>1000 \mu \mathrm{g} / \mathrm{ml}$ ), e.g., SK501 (table II), may carry a plasmid such as pSK11 (Skurray et al., 1988). This plasmid is related to the pSK 1 family of plasmids, but possesses an additional copy of $\operatorname{Tn} 4002$, a transposon specifying $\beta$-lactamase production, which has inserted within the $\mathrm{Ac}^{\mathrm{r}} \mathrm{Eb}^{\mathrm{r}} \mathrm{Qa}^{\mathrm{r}}$ gene, thereby abolishing this resistance phenotype (Gillespie et al., 1988). Low-level $\mathrm{Tp}^{\mathrm{r}}$ strains (MIC $<100 \mu \mathrm{g} / \mathrm{ml}$ ), e.g., SK457 (table II), carry a chromosomal copy of the $\mathrm{Gm}^{\mathrm{r}}$ transposon Tn4001 as established by DNA hybridisation analysis (Gillespie et al., 1987b). Thus the lack of plasmid-associated phenotypes, such as $\mathrm{Ac}^{\mathrm{r}} \mathrm{Eb}^{\mathrm{r}} \mathrm{Qa}^{\mathrm{r}}$ or high-level $\mathrm{Tp}^{\mathrm{r}}$, indicates a chromosomal location for the $\mathrm{Gm}^{\mathrm{r}}$ determinant, and in the 34 strains examined which demonstrate this phenotype this has held true. The low-level $\mathrm{Tp}^{\mathrm{r}}$ determinant in these and all contemporary methicillin-resistant $S$. aureus strains is chromosomally-encoded and is unrelated to the plasmid-encoded $\mathrm{Tp}^{\mathrm{r}}$ determinant dfrA (Young et al., 1987; Tennent et al., 1988; Rouch et al., 1989).

Strains with high-level resistance to acriflavine, ethidium bromide and to quaternary ammonium compounds

Two related determinants encoding high-level $\mathrm{Ac}^{\mathrm{r}} \mathrm{Eb}^{\mathrm{r}} \mathrm{Qa}^{\mathrm{r}}$ are present in $S$. aureus strains. The first determinant, qac $A$, additionally encodes resistance to the diamidines $4^{\prime} 4^{\prime}$-diamidinodiphenylamine dihydrochloride (Dd) and propamidine isethionate (Pi) (Lyon and Skurray, 1987; Tennent et al., 1989). This determinant is predominantly carried by pSK1-family plasmids but has been detected on a heavy-metal resistance plasmid, pSK57 (Gillespie et al., 1986a). Furthermore, it is entirely likely that chromosomal resistance to $\mathrm{Qa}$ now reported in some isolates (Rahman et al., 1988) results from the integration of a pSK1-family plasmid into the $S$. aureus chromosome (Gillespie et al., 1989). The second determinant, $q a c B$, does not confer resistance to $\mathrm{Dd}$ or Pi (Lyon and Skurray, 1987). qacB has only been found on heavy-metal resistance plasmids in contemporary isolates (e.g., pSK21 and pSK23 in the isolates SK413 and SK654, respectively; tables I and II), but was encoded by plasmids of unknown family origin in isolates from 1973 to 1975 and as early as 1950 . Apart from the minor phenotypic differences of these determinants, qac $A$ and $q a c B$ share substantial restriction map identity and DNA homology (unpublished results).

High-level $\mathrm{Ac}^{\mathrm{r}} \mathrm{Eb}^{\mathrm{r}} \mathrm{Qa}^{\mathrm{r}}$ (qacA) strains may be subdivided by their level of resistance towards the aminoglycoside Gm. Strains susceptible to $\mathrm{Gm}$ carry a pSK1-type plasmid which does not carry the $\mathrm{Gm}^{\mathrm{r}} \mathrm{Tm}^{\mathrm{r}} \mathrm{Km}^{\mathrm{r}}$ transposon $\mathrm{Tn} 4001$. The only pSK1-type plasmid detected in a $\mathrm{Gm}^{\mathrm{s}}$ host has been pSK 18 (SK 430; table II); this plasmid additionally lacks the $\mathrm{Tp}^{\mathrm{r}}$ determinant $d$ fr $A$ and consequently strains harbouring this plasmid demonstrate lowlevel Tp ${ }^{\mathrm{r}}$. High-level $\mathrm{Gm}^{\mathrm{r}}$ strains (MIC $>4096 \mu \mathrm{g}$ / $\mathrm{ml}$ ) are additionally resistant to high levels of $\mathrm{Tm}$, $\mathrm{Km}, \mathrm{Nm}$ and $\mathrm{Ak}$ and carry the pSK1-related plasmid pSK7 (e.g., SK460; table II and figure). pSK7 is structurally identical to pSK 1 except that it lacks Tn4001 and hence the aminoglycoside resistance exhibited by such strains must be chromosomally-encoded. The only distinguishable difference between SK460 and other high-level $\mathrm{Gm}^{\mathrm{r}}$ strains (e.g., SK605, see above) was the carriage of pSK7. Presumably, this difference reflects the loss of such a plasmid from a host, such as SK460, thereby constructing plasmid-less progeny such as SK605.

In our studies, the majority of Australian strains that were resistant to $\mathrm{Gm}$ conferred low-level $\mathrm{Gm}^{\mathrm{r}} \mathrm{Tm}^{\mathrm{r}} \mathrm{Km}^{\mathrm{r}}(97 \%)$ and in $77 \%$ of these, resistance was plasmid-mediated. These low-level $\mathrm{Gm}^{\mathrm{r}}$ strains can be divided into two groups based on their susceptibility to $\mathrm{Tp}$. Strains which demonstrate low-level $\mathrm{Tp}^{\mathrm{r}}$ carry one of three plasmid types, e.g., SK604 (pSK9), SK 565 (pSK 15) and SK656 (pSK 14) (tables I and II and figure), as differentiated by restriction endonuclease analysis. All three plasmids are deleted for the $\mathrm{Tp}^{\mathrm{r}}$ determinant, $d f r A$, and pSK 15 carries a single insertion of Tn 4002 but is otherwise identical to pSK 14. High-level $\mathrm{Tp}^{\mathrm{r}}$ strains comprise those that carry the prototype plasmid, pSK1, such as SK529 (table II and figure), or otherwise identical plasmids that carry a copy of Tn4002 where the insert does not abolish any function of pSK1, e.g., SK456 (pSK4), SK480 (pSK8) and SK634 (pSK16). These Tn4002-harbouring plasmids differ from one another by the locality or orientation of insertion of $\operatorname{Tn} 4002$ in the pSK 1 vector as determined by restriction endonuclease analysis. Since Tn4002 or a Tn4002-like 
element preferentially occupies a chromosomal site in methicillin-resistant $S$. aureus, (Gillespie et al., 1988) the occurrence of this transposon at a secondary site, particularly on a plasmid whose copy number is more than one per bacterial chromosome, may result in increased expression of $\beta$-lactamase production (i.e., effectively increasing the host's MIC towards penicillin). However, no differential MIC was observed between strains that exhibited a single chromosomal copy of Tn4002 compared with strains that possessed a chromosomal copy together with a copy of Tn4002 on a pSK1-family plasmid. Thus, strains bearing plasmids such as pSK 4, pSK 8 or pSK 16 could not be differentiated from pSK 1-harbouring isolates.

Since the division of these strains is based solely on their plasmid-encoded phenotypes, the loss of a plasmid from a host dramatically affects this typing system. For instance, if a strain such as SK529 spontaneously lost the plasmid pSK1, the strain would no longer demonstrate the pSK1-encoded phenotypes-resistance to $\mathrm{Gm}, \mathrm{Tm}, \mathrm{Km}, \mathrm{Ac}, \mathrm{Eb}$, Qa and Tp-and would be typed as a SK20-type strain (see figure). We have observed the loss of this plasmid from strain SK529 and by this typing scheme the resultant derivative strain was typed as SK20. Furthermore, loss of the pSK1-family plasmids pSK4, pSK8, pSK9, pSK 14 and pSK 16 from the strains SK456, SK480, SK604, SK656 and SK634, respectively, resulted in derivative strains with a phenotype equivalent to SK20 (see figure); such a result helped to verify this typing scheme.

\section{Strains with low-level resistance to ethidium bromide and to quarternary ammonium compounds}

The qacC determinant encoding low-level resistance to $\mathrm{Eb}$ and to the quaternary ammonium compounds, Bc and Ct (MICs 30,6 and $4 \mu \mathrm{g} / \mathrm{ml}$, respectively) was originally detected on a $2 \cdot 4-\mathrm{kb}$ plasmid, pSK89, from a methicillin-sensitive strain isolated in 1960 (table II; Lyon and Skurray, 1987). qacC shared DNA homology with the $\mathrm{Eb}^{\mathrm{r}} \mathrm{Qa}^{\mathrm{r}}$ determinant on a small molecular weight plasmid from $S$. epidermidis and with the qacD determinant on self-transmissible aminoglycoside resistance plasmids isolated from $S$. aureus and $S$. epidermidis strains from North America which conferred an identical resistance phenotype (e.g., SK445, harbouring pUW3626; table II and figure; M. T. Gillespie, J. M. Tennent and R. A. Skurray, unpublished results). Since the small molecular weight $\mathrm{Eb}^{\mathrm{r}} \mathrm{Qa}^{\mathrm{r}}$ plasmids have been detected only in methicillin-sensitive strains, low-level $\mathrm{Eb}^{\mathrm{r}} \mathrm{Qa}^{\mathrm{r}}$ is considered to be indicative of a self-transmissible plasmid in methicillin-resistant strains. To verify the involvement of a self-transmissible plasmid in mediating this resistance phenotype, the strains can be tested for resistance towards $\mathrm{Nm}$ and paromomycin $(\mathrm{Pm})$; both antibiotics, along with Ak and $\mathrm{Km}$ are inactivated by the 4'adenyltransferase encoded by members of the self-transmissible plasmid family (Lyon et al., 1987).

\section{Other useful phenotypes for strain identification}

In addition to the phenotypes used within this scheme, other antibiotic resistance phenotypes may be encountered and may be of value in differentiating local strains. Chloramphenicol $(\mathrm{Cm})$ resistance is mediated exclusively by a diverse family of plasmids of $3.5-5.5 \mathrm{~kb}$ in size (Gillespie and Skurray, 1988). These plasmids may belong to one of a number of different incompatibility groups and, therefore, could co-exist with pSK1-family, heavy-metal resistance family or self-transmissibletype plasmids, the former two belonging to incompatibility group 1 (Lyon and Skurray, 1987). It should be noted, however, that $\mathrm{Cm}^{\mathrm{r}}$ is an unstable phenotype as $\mathrm{Cm}^{\mathrm{r}}$ plasmids can be easily lost from a strain when grown in the absence of $\mathrm{Cm}$; this effect is commonly seen in the laboratory and has also been observed in the clinical environment (Fisher, 1960). Resistance to erythromycin (Em) and clindamycin $(\mathrm{Cl})$ is chromosomally-determined, most probably via Tn554 or a related element since strains that display these resistances are also resistant to spectinomycin (Sp; table II) (Murphy et al., 1985). This phenotype is commonly encountered in Australian $S$. aureus strains. Other chromosomally-encoded phenotypes include resistance towards fusidic acid and rifampicin, both of which were rarely detected in $S$. aureus strains, and resistance to the fluoroquinolones (Lyon et al., 1983; Ubukata et al., 1989).

\section{Conclusions}

Traditional methods of typing have failed to differentiate the strains of methicillin-resistant $S$. aureus isolated from Australian hospitals over the last decade. All Australian-type isolates were either non-typable, or only weakly typable at $100 \times$ RTD with bacteriophages of the International Typing Set (Lyon et al., 1984a), although some differentiation of Australian strains has been achieved with experimental bacteriophages (Vickery et al., 1983). Biochemical analyses have also failed to discriminate Australian-type methicillin-resistant $S$. aureus strains since they possess similar biochemical 
properties (Gedney and Lacey, 1982). Plasmid DNA analysis has shown that some Australian hospitals harbour particular plasmid-bearing strains and this has provided limited epidemiological data (Lyon et al., 1984a). By use of the scheme described here we have been able to differentiate these particular plasmid-bearing strains from other hospital isolates, and trace the spread of methicillinresistant $S$. aureus within a given hospital, between different hospitals of one city and between hospitals of different cities. The identity of these strains was subsequently confirmed by plasmid DNA analysis and bacteriophage typing. The full potential of plasmid typing procedures, however, is not realised until plasmids are fingerprinted by restriction endonucleases and the results linked to phenotypic data.

In summary, the phenotypic typing scheme we describe is easily performed in a routine clinical laboratory since it only requires media plates and

\section{REFERENCES}

Coleman D C et al. 1985 Susceptibility to antimicrobial agents and analysis of plasmids in gentamicin- and methicillinresistant Staphylococcus aureus from Dublin hospitals. Journal of Medical Microbiology 20: 157-167.

Cohen M L, Wong E S, Falkow S 1982 Common R-plasmids in Staphylococcus aureus and Staphylococcus epidermidis during a nosocomial Staphylococcus aureus outbreak. Antimicrobial Agents and Chemotherapy 21 : 210-215.

Cookson B D, Phillips I 1988 Epidemic methicillin-resistant Staphylococcus aureus. Journal of Antimicrobial Chemotherapy 21 Suppl C: $57-65$.

Fisher M W 1960 The susceptibility of staphylococci to chloramphenicol: a survey of experimental and clinical experiences. Archives of Internal Medicine 105: 413-423.

Gaston M A et al. 1988 Evaluation of electrophoretic methods for typing methicillin-resistant Staphylococcus aureus. Journal of Medical Microbiology 26: 189-197.

Gedney J, Lacey R W 1982 Properties of methicillin-resistant staphylococci now endemic in Australia. Medical Journal of Australia 1 : 448-450.

Gillespie M T, Lyon B R, Loo L S L, Matthews P R, Stewart P $\mathrm{R}$, Skurray R A $1987 a$ Homologous direct repeat sequences associated with mercury, methicillin, tetracycline and trimethoprim resistance determinants in Staphylococcus aureus. FEMS Microbiology Letters 43, 165-171.

Gillespie M T, Lyon B R, Messerotti L J, Skurray R A 1987 b Chromosome-and plasmid-mediated gentamicin resistance in Staphylococcus aureus encoded by Tn4001. Journal of Medical Microbiology 24: 139-144.

Gillespie M T, Lyon B R, Skurray R A 1988 Structural and evolutionary relationships of $\beta$-lactamase transposons from Staphylococcus aureus. Journal of General Microbiology 134: 2857-2866.

Gillespie M T, Lyon B R, Skurray R A 1989 Gentamicin and antiseptic resistance in epidemic methicillin-resistant Staphylococcus aureus. Lancet 1 : 503.

Gillespie M T, May J W, Skurray R A 1984 Antibiotic susceptibilities and plasmid profiles of nosocomial methi- in that respect is far less expensive and less labour intensive than procedures employing ${ }^{35} \mathrm{~S}$-protein analysis or immunoblotting. It should be noted that Australian methicillin-resistant $S$. aureus have also been reported in London hospitals (Townsend $e t$ al., 1984) and from the characteristics (plasmid sizes and phenotypes and the location of antibiotic resistance determinants) of the EMRSA strain detected in London and South-East London hospitals (Cookson and Phillips, 1988), this strain is also equivalent to the Australian strains we describe here. We hope that this paper will form a useful reference for clinical microbiologists and others attempting to monitor the spread of EMRSA in the UK and for those who wish to devise protocols for the identification of new or endemic strains of $S$. aureus.

This work was supported by a Project Grant from the National Health and Medical Research Council of Australia.

cillin-resistant Staphylococcus aureus : a retrospective study. Journal of Medical Microbiology 17: 295-310.

Gillespie M T, May J W, Skurray R A 1985 Antibiotic resistance in Staphylococcus aureus isolated at an Australian hospital between 1946 and 1981. Journal of Medical Microbiology 19: $137-147$.

Gillespie M T, May J W, Skurray R A $1986 a$ Plasmid-encoded resistance to acriflavine and quaternary ammonium compounds in methicillin-resistant Staphylococcus aureus. FEMS Microbiology Letters 34: 47-51.

Gillespie M T, May J W, Skurray R A $1986 b$ Detection of an integrated tetracycline resistance plasmid in the chromosomes of methicillin-resistant Staphylococcus aureus. Journal of General Microbiology 132: 1723-1728.

Gillespie M T, Skurray R A 1988 Structural relationships among chloramphenicol-resistance plasmids of Staphylococcus aureus. FEMS Microbiology Letters 51 : 205-210.

Kozarsky P E, Rimland D, Terry P M, Wachsmuth K 1986 Plasmid analysis of simultaneous nosocomial outbreaks of methicillin-resistant Staphylococcus aureus. Infection Control 7: 577-581.

Krikler S J, Pennington T H, Petrie D 1986 Typing of strains of Staphylococcus aureus by Western blot analysis of culture supernates. Journal of Medical Microbiology 21 : 169-171.

Lee W, Burnie J P 1988 Fingerprinting methicillin-resistant Staphylococcus aureus by the immunoblot technique. Journal of Medical Microbiology 25: 261-268.

Lunn G, Sansone E B 1987 Ethidium bromide: destruction and decontamination of solutions. Analytical Biochemistry 162: 453-458.

Lyon B R, Gillespie M T, Byrne M E, May J W, Skurray R A 1987 Plasmid-mediated resistance to gentamicin in Staphylococcus aureus: the involvement of a transposon. Journal of Medical Microbiology 23: 101-110.

Lyon B R, Iuorio J L, May J W, Skurray R A 1984a Molecular epidemiology of multiresistant Staphylococcus aureus in Australian hospitals. Journal of Medical Microbiology 17: 79-89.

Lyon B R, May J W, Skurray R A 1983 Analysis of plasmids in nosocomial strains of multiple-antibiotic-resistant Staphy- 
lococcus aureus. Antimicrobial Agents and Chemotherapy 23: 817-826.

Lyon B R, May J W, Skurray R A $1984 b$ Tn400I: a gentamicin and kanamycin resistance transposon in Staphylococcus aureus. Molecular and General Genetics 193: 554-556.

Lyon B R, Skurray R A 1987 Antimicrobial resistance of Staphylococcus aureus: genetic basis. Microbiological Reviews 51 : 88-134.

Matthews P R, Reed K C, Stewart P R 1987 The cloning of chromosomal DNA associated with methicillin and other resistances in Staphylococcus aureus. Journal of General Microbiology 133: 1919-1929.

Murphy E, Huwyler L, Bastos M C F 1985 Transposon Tn554: complete nucleotide sequence and isolation of transposondefective and antibiotic-sensitive mutants. EMBO Journal 4: 3357-3365.

Rahman M, Naidoo J, George R C 1988 New genetic location of gentamicin-resistance in methicillin-resistant Staphylococcus aureus. Lancet 2: 1256.

Rhinehart E et al. 1987 Nosocomial clonal dissemination of methicillin-resistant Staphylococcus aureus: elucidation by plasmid analysis. Archives of Internal Medicine 147: 521524.

Richardson J F, Chittasobhon N, Marples R R 1988 Supplementary phages for the investigation of strains of methicillinresistant Staphylococcus aureus. Journal of Medical Microbiology 25 : 67-74.

Rouch D A, Messerotti L J, Loo L S L, Jackson C A, Skurray R A 1989 Trimethoprim resistance transposon $\mathrm{Tn} 4003$ from Staphylococcus aureus encodes genes for a dihydrofolate reductase and thymidylate synthase flanked by three copies of IS257. Molecular Microbiology 3: 161-175.

Skurray R A et al. 1988 Multiresistant Staphylococcus aureus: genetics and evolution of epidemic Australian strains. Journal of Antimicrobial Chemotherapy 21 Suppl C: 19-39.
Stephenson J R, Crook S J, Tabaqchali S 1986 New method for typing Staphylococcus aureus resistant to methicillin based on sulphur- ${ }^{35}$ methionine labelled proteins: its application in an outbreak. British Medical Journal 293: 581-583.

Tennent J M, Lyon B R, Gillespie M T, May J W, Skurray R A 1985 Cloning and expression of Staphylococcus aureus plasmid-mediated quaternary ammonium resistance in Escherichia coli. Antimicrobial Agents and Chemotherapy 27: 79-83.

Tennent J M, Young H-K, Lyon B R, Amyes S G B, Skurray R A 1988 Trimethoprim resistance determinants encoding a dihydrofolate reductase in clinical isolates of Staphylococcus aureus and coagulase-negative staphylococci. Journal of Medical Microbiology 26 : 67-73.

Tennent J M, Lyon B R, Midgley M, Jones I G, Purewal A S, Skurray R A 1989 Physical and biochemical characterization of the qacA gene encoding antiseptic and disinfectant resistance in Staphylococcus aureus. Journal of General Microbiology 135 : 1-10.

Townsend D E, Ashdown N, Bradley J M, Pearman J W, Grubb W B 1984 “Australian” methicillin-resistant Staphylococcus aureus in a London hospital? Medical Journal of Australia 141 : 339-340.

Ubukata K, Itoh-Yamashita N, Konno M 1989 Cloning and expression of the nor $A$ gene for fluoroquinolone resistance in Staphylococcus aureus. Antimicrobial Agents and Chemotherapy 33: 1535-1539.

Vickery A M, Beard-Pegler M A, Rountree P M 1983 Strain differentiation in methicillin-resistant Staphylococcus aureus. Pathology 15 : 235-240.

Young H-K, Skurray R A, Amyes S G B 1987 Plasmid-mediated trimethoprim-resistance in Staphylococcus aureus: characterization of the first gram-positive plasmid dihydrofolate reductase (type S1). Biochemical Journal 243: 309-312. 